\title{
Simulation-based Evaluation of the Diffusion-based Physical Channel in Molecular Nanonetworks
}

\author{
Nora Garralda*, Ignacio Llatser*, Albert Cabellos-Aparicio*, Massimiliano Pierobon ${ }^{\dagger}$ \\ * NaNoNetworking Center in Catalunya (N3Cat) \\ Universitat Politècnica de Catalunya \\ Barcelona, Spain \\ E-mail: \{garralda, llatser, acabello\}@ac.upc.edu \\ $\dagger$ Broadband Wireless Networking Laboratory \\ School of Electrical and Computer Engineering \\ Georgia Institute of Technology, Atlanta, Georgia 30332, USA \\ E-mail: maxp@ece.gatech.edu
}

\begin{abstract}
Nanonetworking is an emerging field of research, where nanotechnology and communication engineering are applied on a common ground. Molecular Communication (MC) is a bio-inspired paradigm, where Nanonetworks, i.e., the interconnection of devices at the nanoscale, are based on the exchange of molecules. Amongst others, diffusion-based MC is expected to be suitable for covering short distances $(\mathrm{nm}-\mu \mathrm{m})$. In this work, we explore the main characteristics of diffusion-based MC through the use of N3Sim, a physical simulation framework for MC. N3Sim allows for the simulation of the physics underlying the diffusion of molecules for different scenarios. Through the N3Sim results, the Linear Time Invariant (LTI) property is proven to be a valid assumption for the free diffusion-based MC scenario. Moreover, diffusion-based noise is observed and evaluated with reference to already proposed stochastic models. The optimal pulse shape for diffusion-based $\mathrm{MC}$ is provided as a result of simulations. Two different pulse-based coding techniques are also compared through N3Sim in terms of available bandwidth and energy consumption for communication.
\end{abstract}

\section{INTRODUCTION}

Nanomachines are the simplest devices in the nanoscale, able to perform tasks such as computing, sensing, information storage and actuation. Nanonetworks, i.e., the interconnection of nanomachines, allow cooperation among them in order to fulfill more complex tasks and increase their operation range, thus expanding their capabilities. Nanonetworks are expected to be applied in many different fields, ranging from the environmental, to the industrial and the biomedical [1].

Amongst other proposed techniques, molecular communication enables the interconnection of nanomachines through the exchange of molecules. Molecular communication spans several different communication solutions according to the way molecules are propagated and they are classified in relation to the communication range. For short distances (nm$\mu \mathrm{m})$, molecular motors [2] and calcium signaling have been proposed [3], [4]; for the medium range ( $\mu \mathrm{m}-\mathrm{mm})$, the use of the flagellated bacteria and catalytic nanomotors [5] has been studied and simulated; for the long range (mm-m), communication by means of pheromones has been suggested [6] as a possible solution.
This work is focused on diffusion-based molecular communication, where information is encoded by the transmitter nanomachine in the molecule release pattern, creating a specific sequence of molecules concentration values at the transmitter location. These changes in the concentration eventually reach a receiver nanomachine, which is placed at a distance from the transmitter. The propagation of the changes in the molecule concentration is due to the diffusion process. The receiver decodes the transmitted information by sensing the local concentration at its location [7].

Diffusion-based molecular communication, and the resulting molecular nanonetworks, are especially aimed to intrabody scenarios due to their low power requirements and high biocompatibility. Potential applications of molecular nanonetworks include, but are not limited to, intelligent drug delivery systems, diagnostic and therapeutic antiviral activities, and prosthetic implants techniques [8], [9], [10].

Several research efforts have been focused on the physical modeling of the channel in diffusion-based molecular communication. As an example, physical models for the channel as well as bio-inspired transmitters and receivers are provided in [7]. Information-theoretical approaches which compute the channel capacity have also been applied in [11], [12], [13], [14]. We believe that in order to validate these models and provide a common ground to compare them, tests either by means of simulation or experimentation should be provided.

The work presented in this paper is focused on the study of the diffusion-based molecular communication channel by using the simulation framework N3Sim [15]. Through N3Sim we are able to simulate the physics underlying the propagation of molecules in different molecular communication scenarios. As a result, we obtain several parameters related to the communication characteristics of the diffusion-based molecular communication channel, such as the attenuation, the delay and the noise features. N3Sim enables also the comparison between different modulation schemes for the diffusion-based molecular communication.

The remainder of this paper is organized as follows. In 
Section II, we introduce the physical layer simulator for the diffusion-based molecular channel, N3Sim. In Section III, we review the different diffusion scenarios related to the assumptions on the physical behavior of the molecules. In Section IV, we examine the noise in a molecular channel and we validate it by simulation. In Section $\mathrm{V}$, we prove that the diffusion-based molecular communication channel is linear and time-invariant. In Section VI, we show the effects of changing the shape and length of the transmitted pulses. Next, in Section VII, we evaluate several modulation techniques and we find the optimal scheme in terms of communication performance. Finally, Section VIII concludes the paper.

\section{N3SIM OVERVIEW}

N3Sim is a simulation framework for the general case of diffusion-based molecular communication, which simulates the diffusion-based molecular channel according to the Brownian dynamics of the molecules in a fluid medium. Through N3Sim, we also simulate interactions among the molecules, such as elastic collisions, when molecules exchange kinetic energy, or electrostatic interactions that appear when the molecules have an electrical charge. As an extension of the Brownian motion, N3Sim simulates also the correlated random walk.

The user can edit multiple parameters, such as the number of transmitters/receivers (as well as their locations), the radius of emitted molecules, the emission pattern for each transmitter, the fluid viscosity and the diffusion coefficient, and a bounded/unbounded space, amongst others.

Through N3Sim, a user may choose how the transmitter encodes the information: i) into the number of emitted molecules, known as information molecules, or ii) into the molecule concentration at the transmitter location. Furthermore, receivers estimate the local concentration by counting the number of molecules within their sensing area over time. The user may also choose between two types of receiver: i) an ideal receiver transparent to the diffusion process, and ii) a receiver that absorbs the molecules after they enter its actuation area.

The benefits of N3Sim with respect to other diffusion-based molecular communication simulators, [16] and [17], comes from the fact that it simulates the motion of every single molecule independently, which allows for the observation of the effect of the molecules interactions and the uncertainty introduced by the Brownian motion. Moreover, N3Sim allows the simulation of scenarios having virtually any number of transmitters and receivers. This feature enables simulations where the molecular information is broadcast from one transmitter to many receivers, or where more than one transmitter access the channel at the same time.

More details about N3Sim may be found in the website of the NaNoNetworking center in Catalunya [15].

\section{Molecular Diffusion Simulation Scenarios}

The diffusion process is defined as the spontaneous spread of molecules in the space upon a molecule concentration gradient. This process tends towards the homogenization of the molecule concentration in the fluid medium, causing a net flux of molecules from zones with higher concentration to the lower concentration zones [18].

The diffusion process is mathematically modeled by the Fick's laws of diffusion for the case of having a moderate concentration of molecules, so that each molecule motion can be considered as independent [19]. Brownian motion is the continuous-time stochastic process which underlies diffusion, and it is observed in a microscopic scale as the jittery motion that the molecules show upon collision with other neighboring molecules.

Brownian dynamics defines the mean square displacement of a molecule subject to diffusion, in each direction, as $\langle x\rangle^{2}=2 D t^{\alpha}$. $D$ is the diffusion coefficient, defined for spherical molecules whose mass and size are much larger than those of the medium in which they are floating. This case corresponds to low Reynolds number conditions [20], which means that viscous forces dominate the process. When the mean square displacement is directly proportional to time, $\alpha=1$, then the resulting process is called free diffusion. This scenario, can be simulated through N3Sim, which models the motion of every single molecule following the Brownian dynamics, and the results are validated by comparison to the Fick's laws of diffusion.

When $\alpha<1$ or $\alpha>1$, the resulting process is called anomalous diffusion. Collective diffusion is a case of anomalous diffusion and it occurs when there is a very high concentration of molecules. Due to the large amount of molecules, the interactions between them impact the diffusion process, which can no longer be modeled with the Fick's laws of diffusion.

The mathematical process underlying anomalous diffusion is the correlated random walk, a Lèvy process [21] which includes memory. This contrasts with the Brownian motion, whose main feature is the absence of time correlation, thus being a memoryless process. Correlated random walk is a discrete version of the Brownian motion, that spans the whole range of random walks, from ballistic to dispersal motions [22], where molecules always experience the same step size and they change their direction with a certain probability. The macroscopic view of the correlated random walk is modeled by the hyperbolic diffusion equation.

N3Sim allows modeling collective diffusion by simulating a large number of molecules and by taking into account elastic collisions among them. The observed results are that, in the tested scenarios, collisions among the information molecules have the same effect as collisions among the information and the fluid molecules. As a result, in this scenario the Fick's laws are a valid model for the molecule diffusion. Nevertheless, if electrostatic interactions are taken into account, these forces dominate the particle motion, which is then much faster and more directed than in the free diffusion and the Fick's laws are not longer valid. Finally, by adding an initial velocity to the emitted molecules, as well as inertia, which is the property of a molecule to remain at a constant velocity, N3Sim approximates the case of correlated random walk. The results for this case are validated by comparison to the hyperbolic 


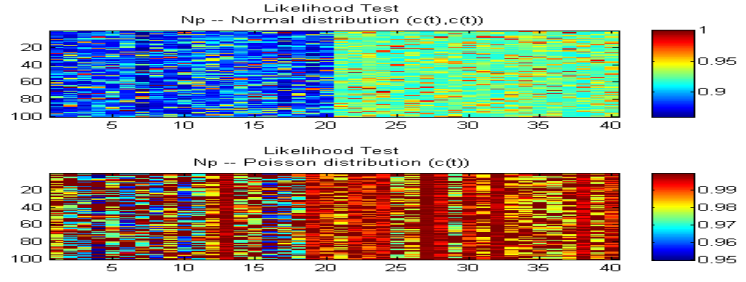

Fig. 1. Normal and Poisson Likelihood tests comparison

diffusion equation solution [7].

All the simulations performed throughout this paper consider the free diffusion scenario, and the space is considered as 2-dimensional.

\section{NoISE IN Diffusion-BASEd Molecular COMMUNICATION}

Brownian motion is a source of uncertainty in the space distribution of particles, and it causes the exact location of a particle to be unknown. As a consequence, even in a system with a homogeneous particle concentration, we cannot predict the exact number of particles within a space area.

When a receiver estimates the concentration by sensing the number of molecules within its actuation area over time, the uncertainty of Brownian motion is observed as a noise source, i.e., an unwanted perturbation on the received concentration signal. This effect has been modeled by means of a probabilistic model in [23], and it is called Particle Counting Noise. In this model, the number of particles $N p$ measured by a receiver follows a non-homogeneous Poisson counting process, whose rate of occurrence is equal to the concentration $c(t)$ of molecules: $N_{p} \sim \operatorname{Poiss}(c(t))$

We have validated the diffusion noise through N3Sim by verifying that the number of particles in a space follows a non-homogeneous Poisson counting process, with the actual concentration as the rate of the process, by the comparison of two likelihood tests.

We consider a scenario with two different homogeneous concentrations of molecules over time. We first simulate the system with the lower concentration and after that we apply the higher one. We have created our data set by locating one hundred receivers all over the system that estimate the concentration independently. Each estimation shows unique values with respect to other estimation performed by other receivers in the same conditions. This is a consequence of the Particle Counting Noise.

In Fig. 1, we can see the result of two likelihood tests. The upper likelihood test shows how likely the number of measured molecules $N p$ is to follow a normal process with mean and variance equal to the actual concentration. The lower likelihood test shows how likely is $N p$ to follow a Poisson process with rate equal to the actual concentration. The mean value of the likelihood tests are 0.91 and 0.9923 , respectively. As a consequence, the simulation results indicate that diffusion-based noise is better modeled by the Particle Counting Noise from [23].

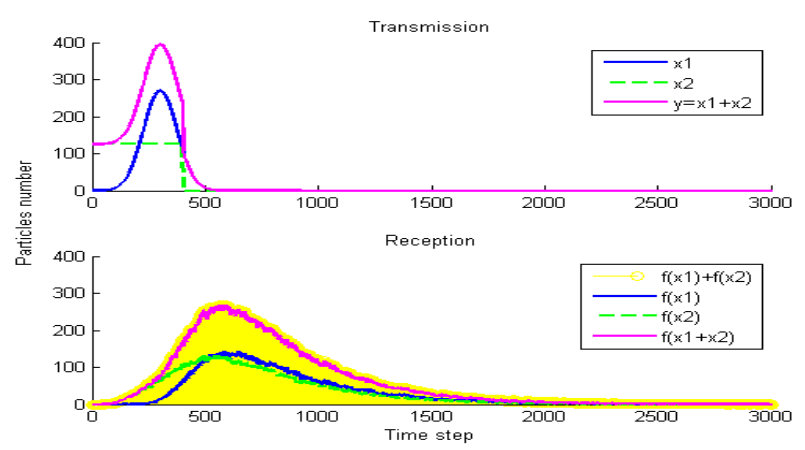

Fig. 2. Linearity validation for the single user scenario by verifying the homogeneity and additive properties. Transmission and reception of a Gaussian pulse, a square pulse and the addition of both.

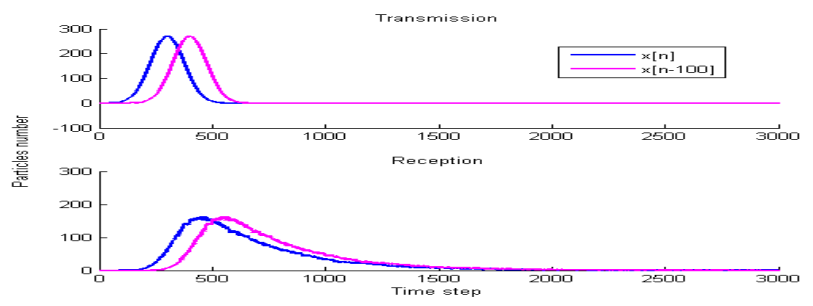

Fig. 3. Temporal invariance validation for the single-transmitter scenario. Transmission and reception of two Gaussian pulses in different times.

This noise can be related to the shot noise in optical communications. Similarly to the shot noise, the Particle Counting Noise, is signal-dependent, i.e., the higher is the power of the transmitted signal, the higher is the power of the noise that affects the received signal.

\section{Channel Linearity And Time Invariance}

A Linear Time-Invariant (LTI) channel fulfills the superposition principle, as well as maintains its features over time. In order to verify whether the molecular diffusion-based channel has the LTI property, we have considered two cases: the singletransmitter scenario and the multi-transmitter scenario. We analyze them in the following:

\section{A. Single-transmitter scenario}

In Fig. 2, the superposition principle is verified. The upper image shows three different transmitted signals: a Gaussian pulse $(x 1)$, a square pulse $(x 2)$, and the addition of both pulses $(y=x 1+x 2)$. The lower image shows the reception of these pulses at a certain distance, as well as the colored area which is the sum of the outputs as if their corresponding inputs where applied independently with respect to the channels. As it is shown in Fig. 2, the colored area matches with the output of the sum of the transmitted signals. These results should confirm the linearity of the channel.

The upper image of Fig. 3 shows the transmitted signals, two Gaussian pulses, one delayed with respect to the other, $(x[n], x[n-100])$. The lower image of Fig. 3 shows the reception of the pulses, and how the delay between the outputs 


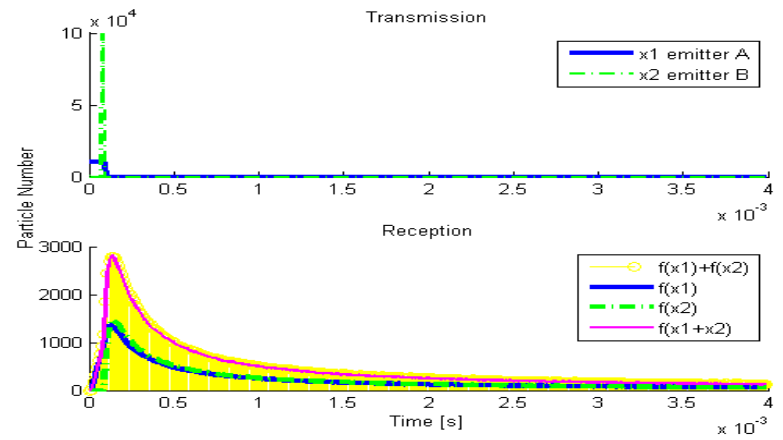

Fig. 4. Linearity validation for the multi-user scenario by verifying the homogeneity and additive properties. Transmission and reception of two square pulses, one wider than the other but with the same transmitted energy.

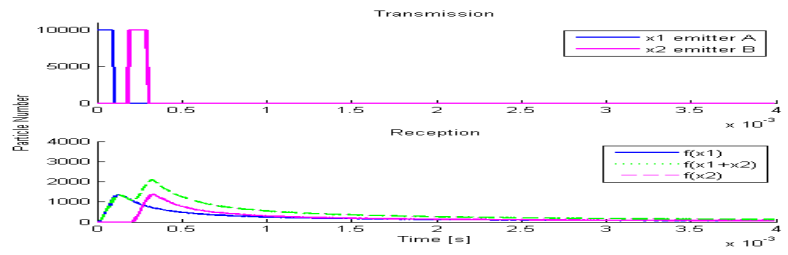

Fig. 5. Temporal invariance validation for the multi-user scenario. Transmission and reception of two square pulses from different emitters, in different times.

matches with the delay between the corresponding inputs. As a consequence, the channel behaves as time-invariant.

\section{B. Multi-transmitter scenario}

For the case of having more than one transmitter, first, transmitter A transmits a pulse, and after that, a second transmitter B transmits another pulse. When both pulses are transmitted at the same time, we check whether an equidistant receiver measures the same signal power as the sum of the two outputs corresponding to single pulses transmitted independently

In Fig. 4, the superposition principle is validated. We observe in the upper image the transmission of two different signals from transmitters $\mathrm{A}$ and $\mathrm{B}$, a square pulse $(x 1)$, and a spike of particles $(x 2)$ as the narrowest possible pulse emitted, both containing the same energy. The lower image shows the reception by a receiver $500 \mathrm{~nm}$ away from both transmitters, $(f(x 1), f(x 2))$, as well as the reception of the simultaneous transmission of the two pulses $(f(x 1+x 2))$. Finally, the colored area shows the sum of the two independent transmissions $(f(x 1)+f(x 2))$. This area matches with the received signal corresponding to the emission of both pulses simultaneously, thus confirming that the channel shows the linearity property also for the multi-transmitter case.

In Fig. 5, we observe that the channel is time-invariant also for the multi-transmitter case. The upper image shows both transmitters, $\mathrm{A}$ and $\mathrm{B}$, transmitting a square pulse in different time instants, $(x 1, x 2)$. The lower image shows the reception of each transmission, $(f(x 1), f(x 2))$ at a distance of $500 \mathrm{~nm}$ from the transmitters, and the received signal when both transmitters are transmitting simultaneously $(f(x 1+x 2))$. Again, we conclude that the delay among the transmitted pulses is the same whether or not they are transmitted simultaneously.

In conclusion, we have obtained that the channel satisfies both conditions of linearity and time invariance for both scenarios, the single-transmitter scenario and the multi-transmitter scenario. This means that when different transmitters transmit the same type of particles, the interactions among them are negligible and the channel can be modeled through the Fick's laws of diffusion.

The operation range of nanomachines is limited by the energy of the transmitted pulses or, equivalently, the total number of emitted particles. Since we have observed that the channel is LTI, the effect of one transmitter releasing a certain number of particles to the medium is equivalent to two transmitters, each releasing half of this quantity. Therefore, by adding emitters that transmit coordinately we can extend the range of the transmitted signal.

If different emitters use different types of particles and the interactions among them are negligible, the channel capacity can also be increased by creating independent channels, e.g., by using the Molecular Division Multiple Access technique proposed in [6].

\section{Pulse Shaping}

Due to their simplicity, the communication techniques based on the exchange of concentration pulses seem to be the most reasonable for diffusion-based molecular communication. In the following, we simulate the transmission of different pulse shapes in order to find the optimal shape for pulse-based modulations.

As Fig. 2, 3, 4 and 5 show, the transmitted pulse shape is distorted due to the diffusion process. For any possible transmitted pulse shape, the receiver gets a pulse with a long tail that follows an exponential decay. The widening in reception of the transmitted pulses increases with the distance, and limits the maximum achievable bandwidth

With the objective of testing the channel behavior for different input pulse shapes, we compare three different shapes: a square pulse, a cosine pulse, and a Gaussian pulse. We consider these shapes due to their differences in the frequency domain; for the same time duration, the Gaussian pulse has its frequency components concentrated around the low frequencies, the square pulse is the more widespread in frequency and the cosine pulse is in between.

We evaluate the suitability of each of these pulses by comparing the widening and the attenuation of the received signal for some distance values. The time duration and total energy of the transmitted pulses are fixed to a common value for all three pulse shapes.

Fig. 6 shows the transmission and reception of the three pulses, for a range of $400 \mathrm{~nm}$. The green vertical lines correspond to the time instants when the received signal has the $50 \%, 60 \%, 70 \%, 80 \%$ and $90 \%$ of the total received energy, from left to right, respectively. We observe that all the received pulses have a long tail and they are therefore distorted, albeit with a different degree of distortion. The 


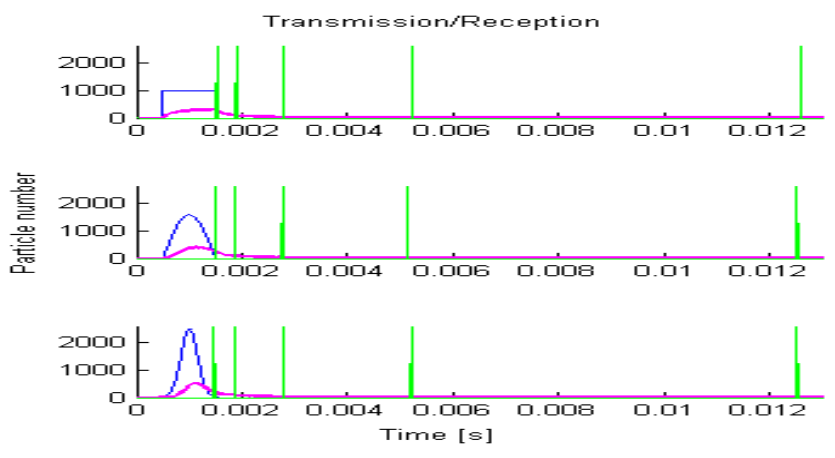

Fig. 6. Square, cosine and Gaussian pulses transmission and its reception by a receiver located $400 \mathrm{~nm}$ away.

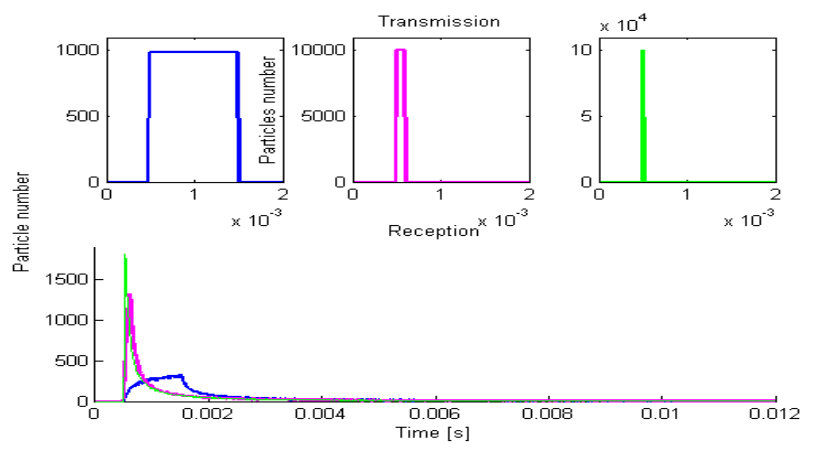

Fig. 7. Transmission and reception of square pulses with the same energy and different time durations.

Gaussian pulse (in the third image) shows the least amount of distortion, while the square one (in the first image) is the most distorted one. The maximum peak level in reception is obtained for the Gaussian pulse. The vertical lines in Fig. 6 show that the energy distribution of the received pulses has almost no dependence on the pulse shape. In conclusion, for a pulse transmission constrained in its energy and in the time duration, the Gaussian shape yields to the best performance, even though the differences with other shapes are minimal.

The widening in the received pulse is distance-dependent. This width determines the maximum achievable bandwidth for this distance. A decrease in the width of the received pulse, and therefore an increase in the achievable bandwidth, can be achieved by transmitting shorter pulses in time. Fig. 7 shows this effect. In the upper side of Fig. 7, the transmitted square pulses are shown, all of them with the same energy but having different lengths. The lower image shows the received pulses for the three transmissions; we observe that the minimum width corresponds to the case of transmitting the shortest square pulse.

After this analysis, we conclude that: i) the optimal pulse shape is a spike, a very narrow pulse, which gives the lowest pulse width at the receiver and thus the highest achievable bandwidth, and ii) the shape of the transmitted pulses is not important, since the total energy received is not shape dependent.

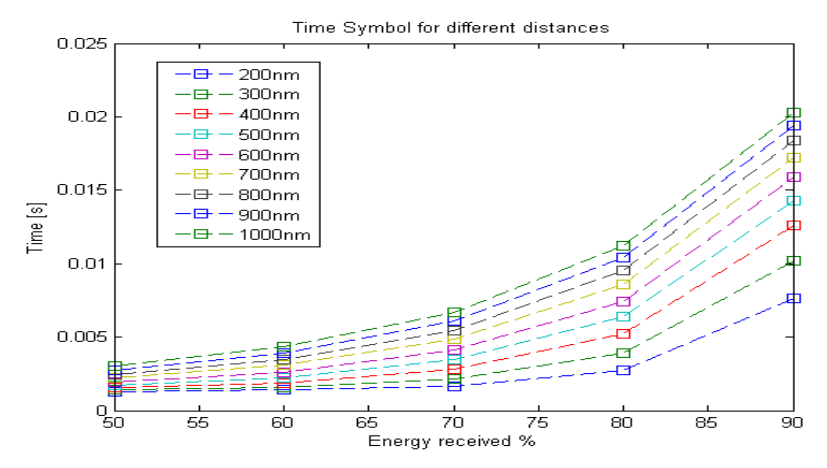

Fig. 8. Symbol duration according to the percentage of received energy for several distances. The transmission distance ranges from $200 \mathrm{~nm}$ to $1000 \mathrm{~nm}$.

\section{Evaluation of Pulse-BAsed Modulation TECHNIQUES}

Communication of larger amounts of information can be achieved by transmitting a train of pulses, and by changing one or more of their parameters. In this section, we evaluate two basic modulation techniques for trains of pulses, namely, the Pulse Amplitude Modulation (PAM), and the Pulse Position Modulation (PPM), which are well-suited to this communication technique, where nanomachines are expected to be very simple devices. We use Gaussian pulse shapes for this analysis.

Any transmitted signal suffers a time widening dependent on the distance between the transmitter and the receiver. In order to avoid Inter-Symbol Interference (ISI), which can create undecodable sequences at the receiver side, a guard time between symbols is added in transmission. The symbol time, $T_{s}$, is the sum of the symbol duration and the guard time.

In this analysis, we use two different symbol times, corresponding to the time instant when the $90 \%$ and the $50 \%$ of the total symbol energy is received, which correspond to $T_{s}=0.02 \mathrm{~s}$ and $T_{s}=0.003$, respectively, for a transmission distance of $1000 \mathrm{~nm}$. Fig. 8 shows how the symbol time increases with the distance.

We consider a particular case of the binary PAM modulation known as On-Off Keying (OOK). In this modulation, a logical " 1 " is transmitted by sending a Gaussian pulse at the beginning of the symbol time, and a logical "0" is transmitted as silence. This scheme is compared with a binary PPM modulation, which requires to divide the symbol time in two halves, and codifies a logical " 1 " by transmitting a pulse during the first half of the symbol time, and a logical " 0 " by transmitting a pulse during the second half.

To evaluate both techniques under the same conditions, the same energy per symbol is transmitted in each of them. This means that, in PPM, the energy of each pulse is half of that in PAM. For this reason, we expect a larger transmission range using PAM. The ISI of the PPM modulation in the case of transmitting a logical "0" followed by a " 1 " is also expected to be higher.

Fig. 9 shows the a comparison of the performance of OOK and PPM. The transmitted sequence is "0110110001". The upper image shows the received signal when the symbol time 


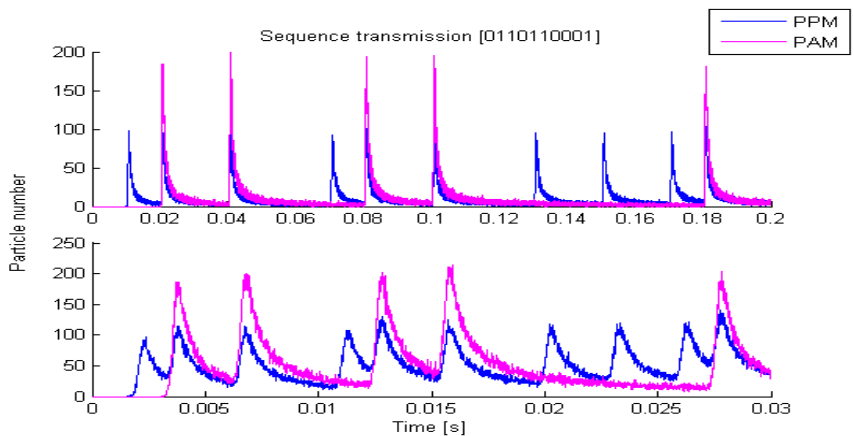

Fig. 9. Comparison of the OOK and PPM modulations for a transmission distance of $1000 \mathrm{~nm}$

corresponds to $90 \%$ of the pulse energy. OOK pulses are received with more energy, thus allowing to cover a larger range in a broadcast communication. The lower image shows the result of the transmission for a shorter symbol time, with similar results. Overall, PPM yields to a higher ISI, as expected.

To summarize, OOK outperforms PPM since the average number of transmitted pulses with $\mathrm{OOK}$ is half of the number of pulses required for PPM. As a consequence, the transmitter can encode the OOK pulses using twice as much energy as with PPM. OOK pulses reach the receiver with a higher power compared to PPM pulses and they are easier to decode. Since in OOK one of the symbols is transmitted as a silence, the ISI is reduced. We conclude that OOK is the most convenient modulation scheme for the molecular diffusion-based channel.

\section{CONCLUSION}

In this work, we detail different diffusion-based scenarios that can be simulated through N3Sim according different assumptions on the physical layer. We have proved the linearity and temporal invariance of the diffusion-based molecular communication channel in the case of free diffusion, by simulating the motion of each particle with Brownian dynamics as well as by including elastic collisions as interactions. We have validated the diffusion-noise when the receiver estimates the concentration by counting the number of molecules in its sensing area, with the Particle Counting Noise model from [23].

After a pulse shaping analysis, we conclude that, the transmission of the shortest possible pulses is needed to reach a given distance range while having the maximum possible bandwidth available. Ideally, the optimal pulse shape is a spike of particles with zero length. The total transmitted energy for a symbol determines its transmission range, and this does not depend on the shape of the pulse. After the comparison of two basic pulse-based communication schemes, we conclude that OOK is the most efficient binary modulation scheme for the diffusion-based molecular communication channel.

We believe that the future work on this topic can take advantage from the results presented in this paper. In particular, these results point out that in an molecular nanonetwork, in oder to minimize the overall energy consumption while allowing for a correct reception of the transmitted signal, the transmitter nanomachines should be able to adjust the energy and the duration of the transmitter pulses. While this can be in contrast with the basic capabilities expected by the nanomachines, we believe that a series of suboptimal schemes can be studied in order to meet possible performance limitations.

\section{REFERENCES}

[1] I. F. Akyildiz, F. Brunetti, and C. Blàzquez, "Nanonetworks: A new communication paradigm," Computer Networks (Elsevier), vol. 52, pp. 2260-2279, 2008.

[2] M. Moore, A. Enomoto, T. Nakano, R. Egashira, T. Suda, A. Kayasuga, H. Kojima, H. Sakakibara, and K. Oiwa, "A Design of a Molecular Communication System for Nanomachines Using Molecular Motors," in IEEE International Conference on Pervasive Computing and Communications, 2006.

[3] J. Q. Liu and T. Nakano, "An information theoretic model of molecular communication based on cellular signaling," in BIONETICS, 2007.

[4] T. Nakano, T. Suda, M. Moore, R. Egashira, A. Enomoto, and K. Arima, "Molecular communication for nanomachines using intercellular calcium signaling," in 5th IEEE Conference on Nanotechnology, vol. 2, pp. 478481.

[5] M. Gregori and I. F. Akyildiz, "A new nanonetwork architecture using flagellated bacteria and catalytic nanomotors," IEEE Journal on Selected Areas in Communications, vol. 28, no. 4, pp. 612-619, 2010.

[6] I. F. Akyildiz and L. Parcerisa, "Molecular Communication Options for Long Range Nanonetworks," Computer Networks (Elsevier), vol. 53, no. 16, pp. 2753-2766, 2009.

[7] M. Pierobon and I. F. Akyildiz, "A physical End-to-End Model for Molecular Communication in Nanonetworks," IEEE Journal on Selected Areas in Communications, vol. 28, no. 4, pp. 602-611, 2010.

[8] G. A. Silva, "Introduction to nanotechnology and its applications to medicine," Surgical Neurology, vol. 61, pp. 216-220, 2004.

[9] R. A. Freitas, "Nanotechnology, nanomedicine and nanosurgery." International Journal of Surgery, vol. 3, no. 4, pp. 243-6, Jan. 2005.

[10] N. H. Malsch, Biomedical Nanotechnology. CRC Press, 2005.

[11] M. Pierobon and I. F. Akyildiz, "Information capacity of diffusionbased molecular communication in nanonetworks," in Proc. of IEEE INFOCOM Miniconference, 2011.

[12] B. Atakan and O. B. Akan, "Deterministic capacity of information flow in molecular nanonetworks," Nano Communication Networks (Elsevier), vol. 1 , no. 1 , pp. 31-42, 2010

[13] — " "On Channel Capacity and Error Compensation in Molecular Communication," Springer Transactions on Computational Systems Biology, vol. 10, pp. 59-80, 2008.

[14] S. Kadloor and R. Adve, "A framework to study the molecular communication system," 2009.

[15] NaNoNetworking Center in Catalunya (N3Cat). [Online]. Available: http://www.n3cat.upc.edu/n3sim/

[16] E. Gul, B. Atakan, and O. B. Akan, "NanoNS: A Nanoscale Network Simulator Framework for Molecular Communications," Nano Communication Networks (Elsevier), vol. 1, no. 2, pp. 138-156, 2010.

[17] M. J. Moore. [Online]. Available: http://www.ics.uci.edu/ mikemo/

[18] J. Philibert, "One and a half century of diffusion: Fick, Einstein, before and beyond," Diffusion Fundamentals, pp. 1.1-1.10, 2005.

[19] T. S. Ursell, "The Diffusion Equation A Multi-dimensional Tutorial," Pasadena, 2007. [Online]. Available: http://www.rpgroup.caltech.edu/ $\sim$ natsirt/aph162/diffusion.pdf

[20] D. L. Ermak and J. A. Mccammon, "Brownian dynamics with hydrodynamic interactions," J. Chem. Phys., vol. 69, pp. 1352-1360, 1978.

[21] L. Vlahos, H. Isliker, Y. Kominis, and K. Hizanidis. (2008, May) Normal and Anomalous Diffusion: A Tutorial. [Online]. Available: http://arxiv.org/abs/0805.0419

[22] V. Méndez, S. Fedotov, and W. Horsthemke, Reaction-Transport Systems: Mesoscopic Foundations, Fronts, and Spatial Instabilities. Springer, 2010.

[23] M. Pierobon and I. F. Akyildiz, "Diffusion-based Noise Analysis for Molecular Communication in Nanonetworks," to appear in IEEE Transactions on Signal Processing, 2011. 